\title{
Vegetación disponible para la producción caprina en el Estado de Hidalgo y su influencia en la calidad de la carne
}

\section{Vegetation Available for Goat Production in Hidalgo State and Its Influence on Meat Quality}

\author{
María F. Chávez Silvestre ${ }^{a}$, Ángel A. Aldrette Arteaga ${ }^{a}$, J. Jesús G. Peralta-Ortiz ${ }^{a}$, Miguel A. \\ Hernández-Zarco ${ }^{b}$, Juan Carlos López-Ojeda ${ }^{b}$ e Isaac Almaraz-Buendía ${ }^{*}$
}

\begin{abstract}
:
The objective of this research consisted of a brief bibliographic investigation about the type of native vegetation capable of being used by goats in the state of Hidalgo. A brief introduction about the situation of goat production worldwide is described at the beginning, then at the national level and later according to the municipalities of Hidalgo. The type of vegetation was related to the consumption habits of the goats and their consumption preferences to finally expose the properties of goat meat.
\end{abstract}

Keywords:

Capra, Herbaceous, Valle del Mezquital, Shrubby

\section{Resumen:}

El objetivo de esta investigación consistió en una breve investigación bibliográfica acerca del tipo de vegetación nativa capaz de ser aprovechada por el ganado caprino en el estado de Hidalgo. Una breve introducción acerca de la situación de la producción caprina a nivel mundial se describe al inicio, luego a nivel nacional y posteriormente acorde a los municipios de Hidalgo. El tipo de vegetación se relacionó con los hábitos de consumo de las cabras y con sus preferencias de consumo para al final exponer las propiedades de la carne caprina.

\section{Palabras Clave:}

Arbustivas, Capra, Herbáceas, Valle del Mezquital

\section{Introducción}

La producción caprina es común en regiones áridas o semi áridas, ya que las cabras son capaces de sobrevivir bajo difíciles condiciones ambientales y aprovechar la vegetación nativa de la región. Las cabras han sido fuente muy importante de proteína animal y leche para poblaciones de países pobres y en desarrollo, además aportan pelo y piel [1].

Los caprinos son animales pequeños con gran agilidad, poseen una velocidad mayor en la formación del bolo alimenticio en comparación a otros rumiantes y un gusto muy desarrollado.

aUniversidad Autónoma del Estado de Hidalgo. María F. Chávez Silvestre, https://orcid.org/0000-0001-6239-6838, Email: ch335817@uaeh.edu.mx; Ángel A. Aldrette Arteaga, https://orcid.org/0000-0003-2892-2165, Email: al317981@uaeh.edu.mx; J. Jesús G. Peralta Ortiz, https://orcid.org/0000-0003-2129-6552, Email: peralta@uaeh.edu.mx; Isaac Almaraz Buendía, https://orcid.org/0000-00019404-1548, Email: isaac_almaraz9974@uaeh.edu.mx.

bColegio de Postgraduados. Miguel A. Hernández Zarco, https://orcid.org/0000-0002-6144-5973, Email: malejandrohdzarco@gmail.com; Juan C. López Ojeda, https://orcid.org/0000-0002-8306-1516, Email: al317981@uaeh.edu.mx

* Autor de Correspondencia: Email: isaac_almaraz9974@uaeh.edu.mx 
Tabla1. Razas de caprinos más comunes en México

\begin{tabular}{c|cc}
\hline Raza & $\begin{array}{c}\text { Peso al } \\
\text { nacimiento } \mathbf{( k g )}\end{array}$ & Fin zootécnico \\
\hline Saanen & 3.67 & Leche \\
Alpina & 3.58 & Leche \\
Toggenburg & 3.56 & Carne \\
Anglo-nubia & 3.28 & Leche \\
Granadina & 2.71 & Carne \\
Boer & 3.07 & Carne \\
Angora & 2.2 & Carne \\
Criolla & Variado & Carne \\
\hline
\end{tabular}

India, China, China Continental, Nigeria, Pakistán, y Bangladesh son los países con mayor inventario caprino [2], y entre ellos poseen el $52 \%$ de la población caprina. Las primeras cabras llegaron a América hace 400 años y se adaptaron rápidamente a las condiciones climáticas de México. Los estados de Oaxaca, Puebla y San Luis Potosí poseen mayor densidad de población caprina, mientras que Zacatecas, San Luis Potosí y Coahuila lideran la producción de carne caprina en canal [3].

La carne de cabra en México representa un gran aporte nutricional, económico y cultural [4]. Por lo anterior, a través de este escrito haremos una breve descripción de la vegetación nativa en el estado de Hidalgo y su relación con la producción caprina.

\section{Materiales y metodos}

La investigación consistió en recopilación bibliográfica en la red mediante Google Académico, Redalyc, SciELO y Biblioteca Digital. Las palabras que se utilizaron en la búsqueda fueron "carne caprina", "producción caprina" "arbustivas" y "forrajes-Hidalgo". La investigación se enfocó tanto en conocer la vegetación nativa disponible para la producción caprina en el Estado de Hidalgo como en identificar las características generales de la producción caprina, donde la búsqueda se restringió a idioma español e inglés del 2000 al 2020 y se restringió acorde a la afinidad con el objetivo planteado.

\section{Resultados y Discusión}

La producción caprina está enfocada hacia la producción de carne y la producción de leche para consumo humano, elaboración de quesos y dulces
[5]. Las principales razas de caprinos en México, su fin zootécnico y peso al nacimiento (Tabla 1) son comunes a través de todo el país debido a que es una especie con inventario limitado comparado con las demás especies de interés pecuario.

Fuente: [4].

Los caprinos son animales muy selectivos que se caracterizan por alimentarse a base de ramoneo y pastoreo [6]. El componente herbáceo y arbustivo contribuye con más del $50 \%$ de la dieta en cabras siendo las hojas las de mayor preferencia. Lo anterior se acentúa más en la estación seca a causa de que muchas hojas permanecen verdes y con buena concentración de nutrientes [7]. Algunas de las arbustivas más palatables son: Atriplex canescens (Costilla de vaca), A. numularia, Mariola, Dalea bicolor (Engorda cabras) [8], Leucaena sp. (Guaje), Pithecellobium dulce (Guamúchil), Acacia farnesiana (Huizache), Guazuma ulmifolia (Guázima), Prosopis juliflora (Mezquite) y Quercus sp. (Encino) [9]. Las leñosas forrajeras son una fuente de forraje muy independiente a la lluvia anual, ya que proveen follaje durante la mayoría del año y aportan frutos de buena calidad, además de hojarasca para el consumo y sombra [7].

En ocasiones se provee un suplemento a base de esquilmos de temporada, por ejemplo, maíz grano (Zeas mays L.), frijol (Phaseolus vulgaris L.), cebada (Hordeum vulgare) y alfalfa (Medicago sativa), siendo administrados principalmente en época de invierno para disminuir el índice de mortalidad. EI suplemento se ofrece antes de salir a pastorear para mejorar la disponibilidad de energía [10].

Producción Caprina y tipo de vegetación en Hidalgo. El estado de Hidalgo representa el $1.1 \%$ de la superficie Nacional y participa con $2.1 \%$ del inventario ganadero nacional caprino [11]. El 9.74\% de la superficie estatal es apta para el aprovechamiento de la vegetación natural por el ganado caprino [12] y se ubica dentro del Valle del Mezquital con base en el informe de la Asociación de Silvicultores de la Región del Valle del Mezquital [13]. El clima semiseco y el templado predominan en la región, donde el primero predomina en mayor proporción. Los municipios que producen el $50 \%$ de la carne caprina en Hidalgo se describen en la Tabla 2, donde Metztitlán, Ixmiquilpan y Zimapán son 
líderes en esta producción pecuaria. En cuanto a la producción de leche caprina, sólo Tecozautla y Nopala de Villagrán se describen en este rubro con 57,189 y 3,928 litros de leche respectivamente en el 2020 [3].

Tabla 2. Producción caprina en Hidalgo

\begin{tabular}{l|cc}
\hline Estado/Municipio & $\begin{array}{c}\text { Producción } \\
\text { (Toneladas) }\end{array}$ & $\begin{array}{c}\text { Carne } \\
\text { (Toneladas) }\end{array}$ \\
\hline Metztitlán & 137.8 & 69.6 \\
Ixmiquilpan & 128.2 & 64.5 \\
Zimapán & 106.1 & 53.2 \\
Cardonal & 83.8 & 42.6 \\
Tasquillo & 51.4 & 26.0 \\
San Agustín & 42.2 & 21.2 \\
Tlaxiaca & & \\
Actopan & 40.8 & 20.6 \\
Zempoala & 40.5 & 20.3 \\
Tula de Allende & 37.6 & 19.1 \\
Tizayuca & 35.7 & 18.1 \\
Villa & 35.5 & 17.9 \\
Tezontepec de & & \\
Tolcayuca & 35.5 & 17.8 \\
\hline
\end{tabular}

Fuente: [3]

En la producción caprina, la vegetación nativa es la fuente de alimentación primaria donde el aprovechamiento de las arbustivas, leñosas y gramíneas no sólo reduce los costos, sino que revitaliza áreas rurales abandonadas [7].

Existe gran variedad de vegetación en Hidalgo, la cual puede ser aprovechada para la producción caprina, ya que gran parte de ésta es la más seleccionada para su consumo. Esta región cuenta con diversos tipos de vegetación dependiendo altitud, topografía y clima. Matorral Desértico Aluvial (predominan Prosopis, Acacia y celtis), Matorral de Prosopis (predomina Prosopis julifora en el estrato arbóreo, yucca filifera y schinus molle en el arbóreo), Matorral de Flourensia, Matorral Crasícaule (predomina Opuntia streptacantha y Prosopis juliflora, Acacia es común en el estrato arbustivo y Agave en el subarbustivo), Matorral de Fouquieria (predomina Acacia sp., Prosopis juliflora), Matorral Desértico Calcicola (predominan Agave sp. y Acacia $s p$.), Matorral de Juniperus (predomina Juniperus fláccida, Opuntis sp., Quercus, sp.), Encinar arbustivo (predomina Quercus sp.) y pastizal inducido (predomina Buchloe dactyloides y Brachiaria meziana) son los principales tipos de vegetación en el Valle del Mezquital [13]. Cabe mencionar que la especie Dalea sp. es común en el Matorral de Fouquieria, Matorral Desértico Calcicola y Encinar arbustivo, lo que sugiere vegetación altamente palatable por las cabras, no obstante, su contenido de nutrientes es variable debido a que la mayoría de las especies arbustivas tienen calidad de baja a moderada. Sin embargo, tienen ventaja sobre las especies herbáceas ya que mantienen su contenido de proteína y minerales durante el periodo de invierno, cuando las herbáceas están inactivas y en primavera-verano cuando están secas y agotadas [14].

El contenido de proteína en la carne caprina es muy similar al de la carne ovina, pero contiene menos grasa intramuscular (Tabla 3) y más cenizas. La proteína animal es muy importante para la nutrición humana por su aporte de aminoácidos.

Tabla 3. Concentración de nutrientes en la carne ovina y caprina

\begin{tabular}{|c|c|c|}
\hline Componente & Caprino & Ovino \\
\hline Cenizas, \% & 4.3 a 4.7 & 3.9 a 4.8 \\
\hline Proteína cruda, \% & 21.5 a 23.5 & 21 a 23.4 \\
\hline $\begin{array}{l}\text { Grasa intramuscular, } \\
\%\end{array}$ & 1.3 a 2.3 & 1.2 a 3.2 \\
\hline $\begin{array}{ll}\text { Ácidos } & \text { grasos } \\
\text { saturados* }^{*} & \end{array}$ & 44.48 & 47.88 \\
\hline $\begin{array}{l}\text { Ácidos grasos } \\
\text { monoinsaturados* }\end{array}$ & 39.56 & 35.12 \\
\hline $\begin{array}{l}\text { Ácidos grasos } \\
\text { poliinsaturados* }\end{array}$ & 16.52 & 18.29 \\
\hline Omega- $6^{*}$ & 12.19 & 12.55 \\
\hline Omega-3* & 4.32 & 5.74 \\
\hline
\end{tabular}

${ }^{*} \mathrm{~g} / 100 \mathrm{~g}$ de lípidos. Fuente: [15]

La carne de cabra es menos jugosa que la carne ovina, pero más oscura, roja y dura [16]. La carne de cabrito no es un alimento que se consuma todos los días, es una carne especialmente cocinada y cara en las zonas donde no se produce. Respecto a los atributos sensoriales de la carne de cabra como la textura, el sabor, el color y el olor, pueden ser afectados por la edad, la raza, la dieta y el sexo del animal, debido a las diferencias en las características biológicas de su 
tejido muscular. Sin embargo, la carne de cabras alimentadas ad libitum tienen un perfil de ácidos grasos más saludable para la salud humana debido a la mayor concentración de ácidos grasos insaturados producto de sus hábitos de ramoneo y consumo de arbustivas y herbáceas [1].

\section{Conclusiones}

Acorde a esta breve revisión bibliográfica, Hidalgo cuenta con una gran variedad de vegetación disponible para el ganado caprino y su consecuente producción de carne. Además, si solo se alimentan ad libitum y con la vegetación del lugar se obtiene carne de calidad y benéfica para la salud humana.

\section{Agradecimientos}

A la Universidad Autónoma del Estado de Hidalgo por las facilidades y el apoyo brindado.

\section{Conflicto de intereses}

Los autores declaran no tener conflictos de interés.

\section{Referencias}

[1] Lopes LS, Martins SR, Chizzotti ML, Busato KC, Oliveira IM Machado OR, Paulino PVR, Lanna DPD, Ladeira MM. Meat quality and fatty acid profile of Brazilian goats subjected to different nutritional treatments. Meat Scince 2014; 97, 602-608.

[2] FAO. FAOSTAT 2021; Recuperado el 10 de junio de 2021, de http://www.fao.org/faostat/en/\#data.

[3] SIAP. Anuario estadístico de la producción ganadera. (G. d. México, Ed.) México, México 2021; Obtenido de https://nube.siap.gob.mx/cierre_pecuario00/.

[4] Secretaría de Agricultura y Desarrollo Rural. La caprinocultura en México. 28 de noviembre de 2017; Obtenido de Gobierno de México: https://www.gob.mx/agricultura/es/articulos/la-caprinocultura-enmexico\#: :text=Las\%20principales\%20razas\%20de\%20cabras,m\%C3 $\% \mathrm{~A} 1 \mathrm{~s} \% 20 \mathrm{de} \% 20160 \% 20$ mil\%20litros.

[5] Tierras Caprino. Producción de caprino en México. MEXICO: VIII FORO NACIONAL DEL CAPRINO; 2017.

[6] Rebollar S. Gastos e ingresos en la actividad caprina extensiva en México. Agronomía Mesoamericana 2012; 23(1).

[7] Papachristou TG, Dziba LE, Provenza FD. Foraging ecology of goats and sheep on wooden rangelands. Small Ruminant Research 2005; 141 156.

[8] Gómez-González A, Pinos-Rodríguez JM, Aguirre-Rivera JR. Manual de Producción Caprina. Universidad Autónoma de San Luis Potosí; 2009.
[9] Camacho L. Effect of season on chemicals composition and in si tu degradability in cows and in adapted and unadapted goats of three Mexican browse species. Animal Feed Science and Technology 2010; 206-212.

[10] Méndoza-Jiménez A, Ortega-Sánchez J. Caracterización de la caprinocultura en el municipio de Tepelmeme Villa de Morelos, Oaxaca, México. Revista Chapingo Serie Zonas Áridas 2009; VIII(2): $75-80$.

[11] SIAP. Hidalgo: Infografía Agroalimentaria 2018. Ciudad de México: Servicio de Información Agroalimentaria y Pesquera 2018. Obtenido de https://nube.siap.gob.mx/gobmx_publicacionnes_siap/pag/2018/Hidalg o-Infografia-Agralimentaria-2018.

[12] INEGI. Anuario estadístico y geográfico de Hidalgo 2017. México: INEGI 2017

[13] Asociación de Silvicultores de la Región del Valle del Mezquital. Estudio Regional Forestal Valle del Mezquital, Hgo. UMAFOR1304 "Valle del Mezquital". Hidalgo: Asociación de Silvicultores de la Región del Valle del Mezquital 2010.

[14] Decandia MY. Foraging Behaviour and Intake of Goats Browsing on Mediterranean Shrublands. En Cannas A. Dairy Goats Feeding and Nutrition (págs. 161 - 182). Italia: CAB International 2008.

[15] Turner k, Belesky D, Cassida K, Zerby H. Carcass merit and meat quality in Suffolk lambs, Katahdin lambs, and meatgoat kids finished on a grass-legume pasture with and without supplementation. Meat Science $2014 ; 1-35$

[16] Suman SP, Joseph P, Li S, Steinke L, Fontaine M. Primary structure of goat myoglobin. Meat Science 2009; 82, 456-460. 